Check for updates

Cite this: RSC Adv., 2017, 7, 39833

Received 17th May 2017

Accepted 13th July 2017

DOI: 10.1039/c7ra05570f

rsc.li/rsc-advances

\title{
Comparative studies on the interaction of nitrofuran antibiotics with bovine serum albumin $\uparrow$
}

\author{
Qiulan Zhang $^{\mathrm{a}}$ and Yongnian Ni (D) *ab
}

The interactions of typical nitrofuran antibiotics, nitrofurazone (NFZ) and nitrofurantoin (NFT), with bovine serum albumin (BSA) have been studied using fluorescence and FT-IR spectroscopy, circular dichroism (CD), atomic force microscopy (AFM) and molecular docking investigation. The results revealed that the fluorescence of BSA was quenched by nitrofurans with a static quenching mechanism. The conformation of BSA was changed and unfolded with the addition of nitrofurans because the alpha-helix and beta-sheet were varied to beta-turn and random structure. This study also indicated that the impact on the conformational changes of BSA caused by NFZ was more conspicuous than that of NFT. The molecular simulation studies were carried out to clearly describe the nature of the interaction between nitrofurans and BSA.

\section{Introduction}

The interactions between biomacromolecules and chemicals have drawn increasing interest in recent years. ${ }^{1-3}$ Serum albumin, a specific biomacromolecule substance, plays important roles in the transport and disposition of different endogenous and exogenous compounds, such as drugs, metabolites, and other biologically active substances, mostly through the formation of noncovalent complexes at typical binding sites, is the major soluble protein in the circulatory system. The ADME (absorption, distribution, metabolism, and excretion) profiles and consequently, the levels of activity and toxicity could be significantly influenced as a result of their binding to serum albumin. ${ }^{4}$ Furthermore, the conformational changes of serum albumin induced by its interaction with small molecular-weight drugs may affect the biological function of serum albumin as the carrier protein. Consequently, investigation of the binding of drugs and their derivatives to serum albumin has great toxicological and medical importance. Hence, the studies of the binding of small molecules with protein will aid the explanation of metabolism and transporting procedure of the interaction and the interpretation of the relationship between the structure and the function of the protein. Being a kind of serum albumin, bovine serum albumin (BSA) has been extensively studied, owing to its structural homology with human serum albumin and the advantages of readily availability, medical importance, and unusual ligand-binding properties. ${ }^{5,6}$

${ }^{a}$ State Key Laboratory of Food Science and Technology, Nanchang University, Nanchang 330047, China. E-mail: ynni@ncu.edu.cn

${ }^{b}$ School of Chemistry, Nanchang University, Nanchang 330031, China

$\uparrow$ Electronic supplementary information (ESI) available. See DOI: 10.1039/c7ra05570f
Nitrofurans, such as nitrofurazone and nitrofurantoin (NFZ and NFT, see formula in Fig. 1), are widely used against common infections, which are pharmaceutically effective as bactericides, schistosomicides, fungicides and amoebicides. ${ }^{7}$ Nitrofurans have been widely used previously in livestock for therapy and precaution of various gastrointestinal infections caused by protozoa or bacteria and also as growth stimulant. ${ }^{8}$ Because of their potentially mutagenic and carcinogenic effects on human health, ${ }^{9}$ the uses of these drugs in food-producing animals are generally prohibited in many countries, except for some special applications. Therefore, the observation and investigation of nitrofurans in feeds may supply an efficient method for filtrating their contamination in feed products, which can result deleterious effect for animals taking on the contaminated materials and subsequently, people expensing products from these food of animal origin. Side-effects with superabundant doses of nitrofuran include central nervous system involvement (excitement, convulsions, tremors, and peripheral neuritis), GI (glycemic index) disturbances, poor weigh gain, and depression of spermatogenesis. Furthermore, different hypersensitivity reactions for human can also be found. ${ }^{10}$ The EU Commission's Decision on March 13, 2003 found the minimum required performance limit (MRPL) of $1.0 \mu \mathrm{g} \mathrm{kg}{ }^{-1}$ for nitrofurans; such as NFZ and NFT, in exporting meat and poultry products. ${ }^{11}$

Although the potential poisonous effects on human health, the uses of nitrofuran antibiotics are still legal in some countries since these compounds are effective veterinaries with relatively low price. The global nitrofuran crisis which broke out during 2002-2015 exposed the frequent presence of the nitrofuran residues in aquaculture and poultry products imported to Europe and America, particularly from some developing countries. ${ }^{12,13}$ 

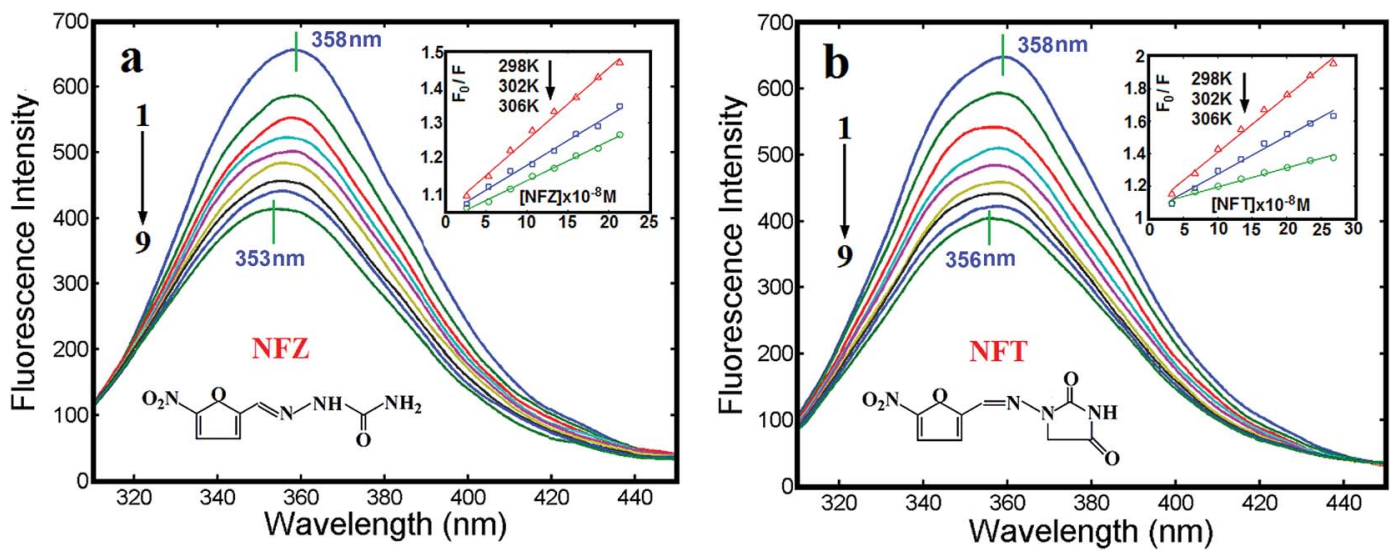

Fig. 1 Fluorescence spectra of BSA in the presence of NFZ/NFT with difference concentration $\left(C_{\mathrm{BSA}}=6.67 \times 10^{-8} \mathrm{~mol} \mathrm{~L}^{-1}, \mathrm{C}_{\mathrm{NFZ} / \mathrm{NFT}}=0,2.67\right.$, $5.34, \ldots, 21.36 \times 10^{-8} \mathrm{~mol} \mathrm{~L}^{-1}$ for curves 1 to 9 , respectively). Insets: Stern-Volmer plots of NFZ/NFT-BSA complexes at 298,302 and $306 \mathrm{~K}$.

A number of analytical methods for trace nitrofuran veterinary residues in the food of animal origin were developed. ${ }^{14,15} \mathrm{In}$ this study, the fluorescence and FT-IR spectroscopy, the CD (circular dichroism) and the AFM (atomic force microscope) were employed to explore the interactions of nitrofurans, FZD or NFT, with BSA in the simulative physiological conditions. The study supplies a quantitative and qualitative understanding of the effect of the nitrofuran antibiotics on the structure and function of BSA, which would be a useful guideline for further understanding the toxicological action for common infections in animals and humans, and also explain its binding mechanism at molecular level.

\section{Experimental}

\subsection{Instruments and reagents}

The fluorescence spectra were measured on a Perkin-Elmer LS55 luminescence spectrometer (Perkin-Elmer Inc., USA) equipped with a thermostatic bath (Model ZC-10, Ningbo Tianheng Instruments Factory, China) and a $1.0 \mathrm{~cm}$ quartz cuvette. The FL Winlab software (Perkin-Elmer) was used to correct the fluorescence data. FT-IR spectra were measured on a Tensor 27 FTIR spectrometer (Bruker, German) equipped with a deuterated triglycine sulfate (DTGS) detector in the rage of $4000-400 \mathrm{~cm}^{-1}$. Circular dichroism (CD) spectra were recorded with a Biologic MOS 450 CD spectrometer (Bio-Logic, France). For measurement in the far-UV (190-280 nm), a quartz cell with a path length of $1.0 \mathrm{~cm}$ was used in nitrogen atmosphere. The atomic force microscopic (AFM) images were acquired by using an AJIII instrument (Shanghai Aijian Nanotechnology, China) with the tapping mode, which also produced simultaneously the intensity and phase data. Standard silicon cantilevers (spring constant: $0.6-6.0 \mathrm{~N} \mathrm{~m}^{-1}$ ) were used at their resonance frequencies (typically $60-150 \mathrm{kHz}$ ). All measurements were carried out at room temperature $\left(25 \pm 0.5{ }^{\circ} \mathrm{C}\right)$ unless stated otherwise.

Stock solutions of $5 \times 10^{-3} \mathrm{~mol} \mathrm{~L}^{-1} \mathrm{NFZ}$ and NFT (Sigma Chemical Co., St. Louis, USA; purity - not less than 99.0\%) were prepared by dissolving their crystals $\left(9.91 \times 10^{-3} \mathrm{~g}\right.$ and $1.19 \times$
$10^{-2} \mathrm{~g}$, respectively) in $10 \mathrm{~mL}$ dimethyl formamide (DMF). BSA $\left(2 \times 10^{-3} \mathrm{~mol} \mathrm{~L}^{-1}\right)$ was prepared by dissolving $1.36 \mathrm{~g}$ of protein $(M=66 \mathrm{kDa}, \geq 98 \%$, lyophilized powder; purchased from Sigma-Aldrich Chemical Co. Ltd., and without further purification) in $10.0 \mathrm{~mL} 5.0 \times 10^{-2} \mathrm{~mol} \mathrm{~L}^{-1}$ sodium chloride solution and stored at $4{ }^{\circ} \mathrm{C}$. To confirm the purity of the prepared BSA, it was diluted to $2.0 \times 10^{-5} \mathrm{~mol} \mathrm{~L}^{-1}$, and the measured absorbance value was (0.896) at $278 \mathrm{~nm}$. This was compared with an absorbance of 0.667 from a reference of $1.0 \mathrm{~g} \mathrm{~L}^{-1}(1.47 \times$ $10^{-5} \mathrm{~mol} \mathrm{~L}^{-1}$ ) pure BSA. ${ }^{16}$ All experimental solutions were adjusted with the Tris-HCl ((hydroxy methyl)amino methanehydrogen chloride) buffer to $\mathrm{pH}$ 7.4. Other chemicals were Analytical grade reagents, and doubly distilled water was used throughout.

\subsection{Fluorescence quenching study}

Solutions used in the fluorescence experiments were prepared in a cuvette with $3.0 \mathrm{~mL}$ of $\mathrm{pH} 7.4$ Tris-HCl buffer containing appropriate amounts of BSA and nitrofurans. BSA was kept at $6.67 \times 10^{-8} \mathrm{~mol} \mathrm{~L}^{-1}, \mathrm{NFZ}$ or NFT, as appropriate, was added to this solution in the range of $0.00-2.13 \times 10^{-7} \mathrm{~mol} \mathrm{~L}^{-1}$ with an interval of $2.67 \times 10^{-8} \mathrm{~mol} \mathrm{~L}^{-1}$ (in such a way, nine samples were obtained), and the total added volume, including the BSA and nitrofurans, to cuvette was less than $0.1 \mathrm{~mL}$ to reduce the volume effect. The well-mixed solutions were allowed to stand for $10 \mathrm{~min}$ at $298 \mathrm{~K}$ before spectroscopic measurements. The excitation and emission slits were set at $10 \mathrm{~nm}$, while the scanning rate was $1500 \mathrm{~nm} \mathrm{~min}{ }^{-1}$. Fluorescence spectra (nine) were then measured in the range of $200-550 \mathrm{~nm}$ at the excitation wavelength of $280 \mathrm{~nm}$. The above fluorescence experiments were repeated at another two temperatures, $302 \mathrm{~K}$ and $306 \mathrm{~K}$, respectively.

\subsection{FT-IR measurements}

All spectra were taken via the attenuated total reflectance (ATR) method with a resolution of $4.0 \mathrm{~cm}^{-1}$ and 60 scans. The FT-IR spectra of BSA $\left(2.5 \times 10^{-6} \mathrm{~mol} \mathrm{~L}^{-1}\right)$ in the absence and presence of nitrofurans were recorded in the range of 
800-2000 $\mathrm{cm}^{-1}$ at $\mathrm{pH} 7.4$ Tris-HCl buffer and room temperature. The molar ratio of nitrofurans to BSA was set to $4: 1 .^{17}$ The corresponding spectra of buffer solution were measured under the same conditions and taken as blank, which were subtracted to obtain the FT-IR spectra of the sample solution. The secondary structure compositions of BSA and its nitrofuran complexes were estimated by applying the curve-fitted process to fit the Gaussian line shapes to the amide I band of the FT-IR spectral data. ${ }^{18}$

\subsection{Circular dichroism (CD) and resonance light scattering (RLS) studies for the interaction}

The CD spectra of BSA $\left(2.5 \times 10^{-7} \mathrm{~mol} \mathrm{~L}^{-1}\right)$ in the presence of nitrofurans were recorded in the range of 200-250 nm under constant nitrogen flush. The molar ratios of nitrofurans to BSA were $4: 1{ }^{19}$ Each of the obtained CD spectrum was an average of three scans and were corrected by the buffer signal. RLS spectra were obtained by synchronous scanning with the excitation and emission monochromators $(\Delta \lambda=0 \mathrm{~nm})$ with a wavelength range of $200-600 \mathrm{~nm}$ on the LS-55 luminescence spectrometer.

\subsection{Atomic force microscopy (AFM) measurements}

Samples for the AFM imaging were prepared by depositing $10 \mu \mathrm{L}$ of BSA, NFZ-BSA and NFT-BSA complexes $(4: 1)$ on freshly cleaved mica plates separately (muscovite mica was cut into about $1.2 \mathrm{~cm} \times 1.2 \mathrm{~cm}$ square pieces as substrate), which were dried overnight.

\subsection{Molecular docking studies}

The 3D structure of NFZ/NFT as ligand was generated in Chem3D Ultra 8.0. The crystal structure of BSA with PDB ID 3VO3 was obtained from Protein data bank (http://www.rcsb.org). The docking simulation was performed with the Lamarckian genetic algorithm for 100 docking runs to find visible combination models using the MGL (molecular graphics laboratory) tools 1.5.6 matches with AutoDock software (version 4.2). ${ }^{20}$

\section{Results and discussion}

\subsection{Fluorescence spectral analysis}

With its non-intrusive and high sensitivity measurement, fluorescence spectroscopy is generally appropriate for low concentration detection under physiological conditions. Usually, the fluorescence emission of BSA is primarily obtained from the residues of tryptophan (Trp), tyrosine (Tyr) and phenylalanine (Phe). In reality, people generally consider the fluorescence of Trp residues as the inherent fluorescence of BSA because of a relatively low quantum yield of phenylalanine and the almost completely quenched of the fluorescence of tyrosine if it is ionized or present near to an amine group, a carboxyl group or a tryptophan. ${ }^{21}$ Fig. 1 shows the fluorescence quenching spectra of BSA induced by NFZ and NFT with different concentrations in Tris-HCl buffer. The fluorescence change indicated the potentially greater toxicity to BSA at higher concentrations of nitrofurans. ${ }^{22,23}$ A blue shift of the emission peak for NFZ-BSA (5 nm, Fig. 1a) and NFT-BSA (2 nm, Fig. 1b) systems occurred, which indicated that the microenvironment around the tryptophan and tyrosine residues of BSA was changed, the polarity around the amino acid residues decreased and the hydrophobicity increased. ${ }^{24}$ Besides, NFZ was seemed to have a greater effect on the structure of BSA than that of NFT.

Static and dynamic quenchings are the main processes of fluorescence quenching. The Stern-Volmer equation has been employed (eqn (1)), ${ }^{25}$ in order to find out the binding mechanism between BSA and nitrofurans:

$$
F_{0} / F=1+K_{\mathrm{q}} \tau_{0}[\mathrm{Q}]=1+K_{\mathrm{Sv}}[\mathrm{Q}]
$$

where $F_{0}$ and $F$ are the steady-state fluorescence intensities without and with quencher nitrofurans, respectively; $K_{\mathrm{q}}$ is the quenching rate constant; $\tau_{0}$ represents the biomolecular average fluorescence lifetime of the molecule in the excited state without any quencher; $K_{\mathrm{SV}}$ is the Stern-Volmer quenching constant and [Q] is the concentration of the quencher. Static quenching is caused by ground-state complex formation among fluorophores. Therefore, increasing temperature will decrease the quenching constant $K_{\mathrm{SV}}$ due to the declined stability of ligand-protein complex in a static quenching system. In constant, dynamic quenching results from collision between a fluorophore and a quencher. ${ }^{26}$ Thus, in a dynamic quenching system, the value of $K_{\mathrm{SV}}$ was increased with increasing temperature. Time-resolved fluorescence (TRF) measurements are sensitive to the microenvironment of fluorophore and are helpful to find excited state interactions. ${ }^{27}$ Unlike steady-state fluorescence emission which is a relative measurement, while the lifetime measurements are generally absolute. See the insets in Fig. 1 , the plot of $F_{0} / F$ versus [Q] was linear, which indicated there was a quenching in the nitrofurans-BSA system. The values of $K_{\mathrm{SV}}$ derived from these plots were 2.01, 1.39, $1.11 \times$ $10^{6} \mathrm{~L} \mathrm{~mol}^{-1}$ and 3.47, 2.34, $1.18 \times 10^{6} \mathrm{~L} \mathrm{~mol}^{-1}$ for the binding of NFZ-BSA and NFT-BSA, respectively, at three different temperatures. Thus, $K_{\mathrm{SV}}$ was decreased gradually with increasing temperature, which suggests that quenching followed a static mechanism and was induced by the formation of complex as shown below:

$$
\mathrm{NFZ/NFT}+\mathrm{BSA} \leftrightarrow \mathrm{NFZ}-\mathrm{BSA} / \mathrm{NFT}-\mathrm{BSA}
$$

The following equation was employed to survey the equilibrium between the free and bound molecules and treat the experimental data on the nitrofuran-BSA systems: ${ }^{24}$

$$
\log \left[\left(F_{0}-F\right) / F\right]=n \log K_{\mathrm{a}}-n \log \left[1 /\left([\mathrm{Q}]-\left(F_{0}-F\right)[\mathrm{P}] / F_{0}\right)\right]
$$

where $F_{0}, F$ and [Q] are the same meaning as in eqn (1) and [P] is the concentration of BSA. According to eqn (3), the values of $K_{\mathrm{a}}$ and $n$ at physiological pH (7.4) could be calculated, and the results at three selected temperatures (298, 302, and $306 \mathrm{~K}$ ) of NFZ and NFT were given in Fig. 2 and Table 1 . The number of binding sites $n$ approximately equated 1 , manifesting that during their binding process there was mere one binding site for nitrofurans in BSA. The gradually decreased $K_{\mathrm{a}}$ indicated the 

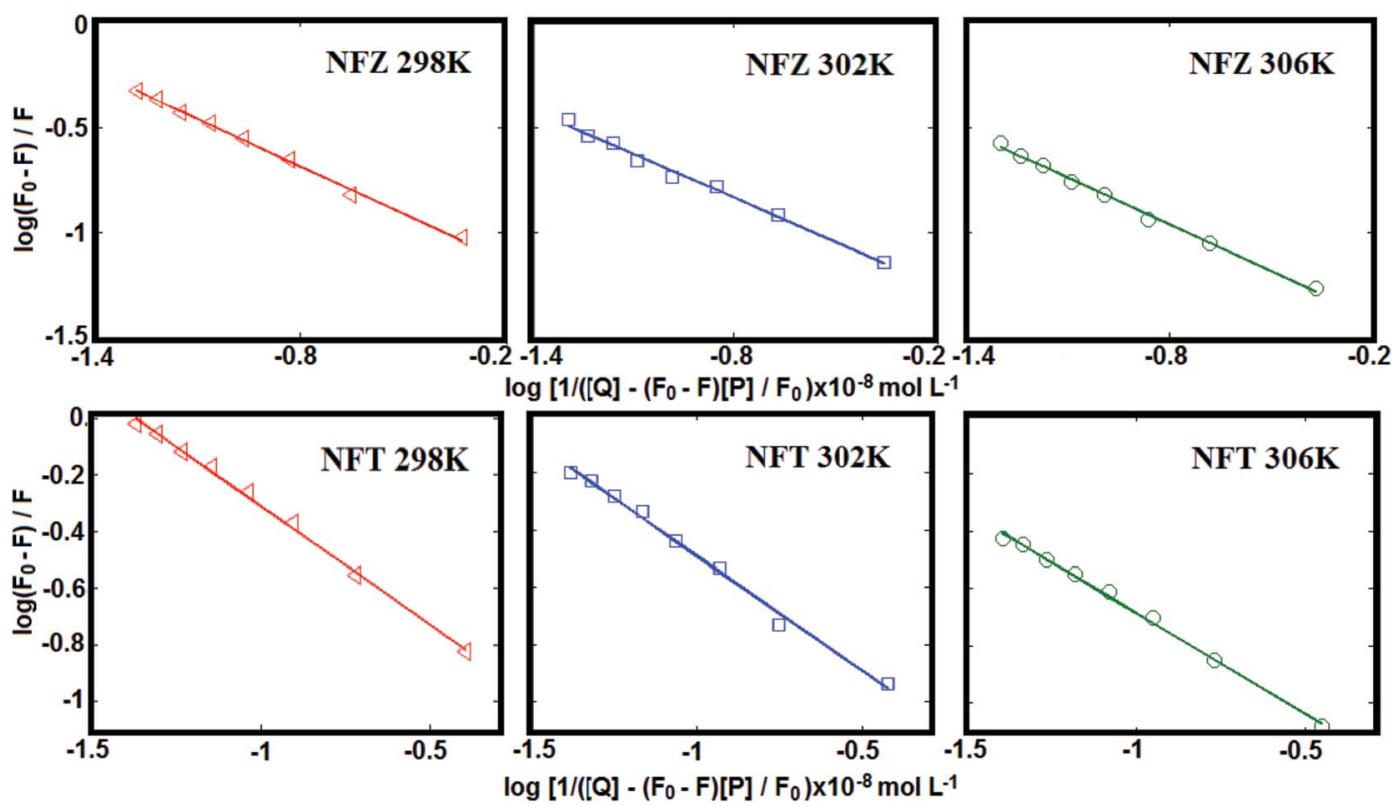

Fig. 2 The plots of $\log \left(F_{0}-F\right) / F$ versus $\log \left[1 /\left([Q]-\left(F_{0}-F\right)[P] / F_{0}\right)\right]$ for the NFZ/NFT-BSA complex systems at 298,302 and $306 \mathrm{~K}$.

instability of the nitrofurans-BSA complex with the raising temperature. The obtained $K_{\mathrm{a}}$ of nitrofurans-BSA system was about $10^{6} \mathrm{~L} \mathrm{~mol}^{-1}$, indicating a high level of binding interaction for nitrofurans-BSA. Furthermore, NFT exhibited a higher affinity to BSA $\left(7.15 \times 10^{6} \mathrm{~L} \mathrm{~mol}^{-1}\right)$, as compared to NFZ $(5.31 \times$ $10^{6} \mathrm{~L} \mathrm{~mol}^{-1}$ ), which could be responsible for their molecular structure. As mentioned above, NFZ has three active N-Hs and is more rapidly metabolized than NFT, which has only one $\mathrm{N}-\mathrm{H}$.

The synchronous fluorescence spectra of BSA in the presence of NFZ/NFT $(\Delta \lambda=60 \mathrm{~nm})$ were shown in Fig. 1S, see ESI. $\dagger$ It was revealed that the fluorescence intensity of BSA was decreased regularly with the increasing addition of NFZ or NFT. Meanwhile, the blue shift ( $3 \mathrm{~nm}$ for NFZ-BSA, and $1 \mathrm{~nm}$ for NFT-BSA) for the emission peaks of tryptophan residues were observed, which indicated that the hydrophobicity surrounding tryptophan residues in BSA increased and the polarity was decreased.

\subsection{Thermodynamic parameters}

The binding forces between ligand and protein include hydrogen bonds, van der Waals interactions, electrostatic forces and hydrophobic interaction forces, which can be determined by the thermodynamic parameters. The enthalpy change $(\Delta H)$ and the entropy change $(\Delta S)$ for the interaction between nitrofurans and BSA were calculated according to the van't Hoff equation (eqn (4)): ${ }^{28}$

$$
\ln K_{\mathrm{a}}=-\frac{\Delta H}{R T}+\frac{\Delta S}{R}
$$

where $K_{\mathrm{a}}$ is the associative binding constant at temperature $T$, and $R$ is the gas constant $\left(8.314 \mathrm{~J} \mathrm{~mol}^{-1} \mathrm{~K}^{-1}\right)$. Moreover, $K_{\mathrm{a}}$ at temperature $T$ could be obtained from the emission quenching, and $\Delta H$ and $\Delta S$ were then obtained from the slope and intercept by plotting $\ln K_{\mathrm{a}}$ to $1 / T$ (i.e. eqn (4)). To elucidate the interaction between nitrofurans and BSA, the thermodynamic parameters were then estimated from the following relationship:

$$
\Delta G=\Delta H-T \Delta S=-R T \ln K_{\mathrm{a}}
$$

As shown in Table 1, the negative $\Delta G$ indicated the spontaneity of the binding interactions between nitrofurans with BSA. $\Delta H$ and $\Delta S$ were found to be $-46.95 \mathrm{~kJ} \mathrm{~mol}^{-1}$ and

\begin{tabular}{|c|c|c|c|c|c|c|c|}
\hline$T(\mathrm{~K})$ & $K_{\mathrm{SV}}\left(\times 10^{6} \mathrm{~L} \mathrm{~mol}^{-1}\right)$ & $R^{2}$ & $K_{\mathrm{a}}\left(\times 10^{6} \mathrm{~L} \mathrm{~mol}^{-1}\right)$ & $n$ & $\Delta G\left(\mathrm{~kJ} \mathrm{~mol}^{-1}\right)$ & $\Delta H\left(\mathrm{~kJ} \mathrm{~mol}^{-1}\right)$ & $\Delta S\left(\mathrm{~J} \mathrm{~mol}^{-1} \mathrm{~K}^{-1}\right)$ \\
\hline \multicolumn{8}{|l|}{ NFZ } \\
\hline 298 & 2.01 & 0.9969 & 5.31 & 0.7376 & $-38.37 \pm 0.12$ & $-46.95 \pm 0.23$ & $-29.20 \pm 0.81$ \\
\hline 306 & 1.11 & 0.9980 & 2.80 & 0.7375 & $-37.77 \pm 0.32$ & & \\
\hline \multicolumn{8}{|l|}{ NFT } \\
\hline 306 & 1.18 & 0.9927 & 4.08 & 0.7044 & $-38.73 \pm 0.29$ & & \\
\hline
\end{tabular}

Table 1 The binding parameters and thermodynamic parameters of the NFZ/NFT and BSA mixture 
$-29.20 \mathrm{~J} \mathrm{~mol}^{-1} \mathrm{~K}^{-1}$ for NFZ-BSA, $-63.57 \mathrm{~kJ} \mathrm{~mol}^{-1}$ and $-81.80 \mathrm{~J} \mathrm{~mol}^{-1} \mathrm{~K}^{-1}$ for NFT-BSA, respectively. The negative $\Delta H$ and $\Delta S$ indicated that hydrogen bonds and van der Waals interactions played a major role in the binding between NFZ/NFT and BSA quenching process.

\subsection{FT-IR spectra of nitrofurans-BSA complexes}

The infrared spectra of proteins are primarily constituted of amide I (1600-1700 $\left.\mathrm{cm}^{-1}\right)$ and amide II (1500-1600 $\left.\mathrm{cm}^{-1}\right)$, which are induced by the carbonyl stretching vibrations of the peptide backbone (amide I band) and the combination of $\mathrm{N}-\mathrm{H}$ in-plane bending and $\mathrm{C}-\mathrm{N}$ stretching vibrations of peptides groups (amide II band). ${ }^{29}$ Generally, the amide I band is more susceptive to change in protein secondary structure than the amide II band. The form of the amide I band is depended on the different secondary structure components, such as $\alpha$-helix (1650-1660 $\left.\mathrm{cm}^{-1}\right), \quad \beta$-sheet $\quad\left(1610-1640 \mathrm{~cm}^{-1}\right), \quad \beta$-turn (1660-1680 $\left.\mathrm{cm}^{-1}\right), \quad \beta$-antiparallel (1680-1690 $\mathrm{cm}^{-1}$ ) and random coil (1641-1648 $\left.\mathrm{cm}^{-1}\right) \cdot{ }^{30}$ Conformational changes of proteins are generally occurred during ligand-protein compound formation procedure. The infrared self-deconvolution with second derivative resolution enhancement and curve-fitting procedures could be applied to determine the secondary structure of BSA. Therefore, the interaction between nitrofurans and BSA was represented by FT-IR spectroscopy. In Fig. 3a, the peak position (the amide I band) was shift from 1655 to 1648 (NFZ) and 1650 (NFT) $\mathrm{cm}^{-1}$, and that of amide II band was shift from 1541 to 1634 (NFZ) and 1635 (NFT) $\mathrm{cm}^{-1}$ along with the changed peak intensity and peak shape in accordance with the addition of NFZ and NFT to BSA. The result indicated that NFZ and NFT
Table 2 The analysis of secondary structure for free BSA and its nitrofuran complexes at $\mathrm{pH} 7.4$

\begin{tabular}{llllll}
\hline Compound & $\begin{array}{l}\alpha \text {-Helix } \\
(\%)\end{array}$ & $\begin{array}{l}\beta \text {-Sheet } \\
(\%)\end{array}$ & $\begin{array}{l}\beta \text {-Turn } \\
(\%)\end{array}$ & $\begin{array}{l}\beta \text {-Anti } \\
(\%)\end{array}$ & $\begin{array}{l}\text { Random } \\
\text { coil }(\%)\end{array}$ \\
\hline BSA & 46.97 & 11.74 & 15.91 & 8.33 & 17.04 \\
NFZ-BSA & 35.94 & 9.38 & 21.88 & 14.06 & 18.75 \\
NFT-BSA & 40.40 & 9.76 & 18.86 & 12.79 & 18.18
\end{tabular}

interacted with the $\mathrm{C}=\mathrm{O}$ and $\mathrm{C}-\mathrm{N}$ groups of the protein polypeptides and led to the reset of the polypeptides carbonyl hydrogen-bonding network. ${ }^{31}$

The quantitative analysis of the free BSA secondary structure and its nitrofurans compounds has been calculated and shown in Fig. $3 \mathrm{~b}-\mathrm{d}$ and Table 2 . The $\alpha$-helix of free protein was $46.97 \%$ $\left(1656 \mathrm{~cm}^{-1}\right.$ ), $\beta$-sheet was $11.74 \%$ (1634 and $1625 \mathrm{~cm}^{-1}$ ), $\beta$-turn structure was $15.91 \%\left(1668 \mathrm{~cm}^{-1}\right), \beta$-antiparallel was $8.33 \%$ (1692 and $1682 \mathrm{~cm}^{-1}$ ), and random coil was $17.04 \%$ $\left(1642 \mathrm{~cm}^{-1}\right)$. The results agreed well with the spectroscopic studies of BSA previously reported. ${ }^{32}$ Interaction with nitrofurans, an obvious decrease of $\alpha$-helix from $46.97 \%$ (free BSA) to $35.94 \%$ (NFZ-BSA) and $40.40 \%$ (NFT-BSA) with a decrease in $\beta$-sheet from $11.74 \%$ (free BSA) to $9.38 \%$ (NFZ), 9.76\% (NFT) was observed. An increase was measured for the $\beta$-turn structure from $15.91 \%$ (free BSA) to $21.88 \%$ (NFZ), $18.86 \%$ (NFT). Similarly, an analogical increase was also surveyed for the $\beta$-anti and random coil for NFZ-BSA and NFT-BSA. The results are also accordance with the reduction in the intensity of the protein amide I band which discussed above. The major decrease in $\alpha$-helix and $\beta$-sheet structure and increase in $\beta$-turn and $\beta$-anti
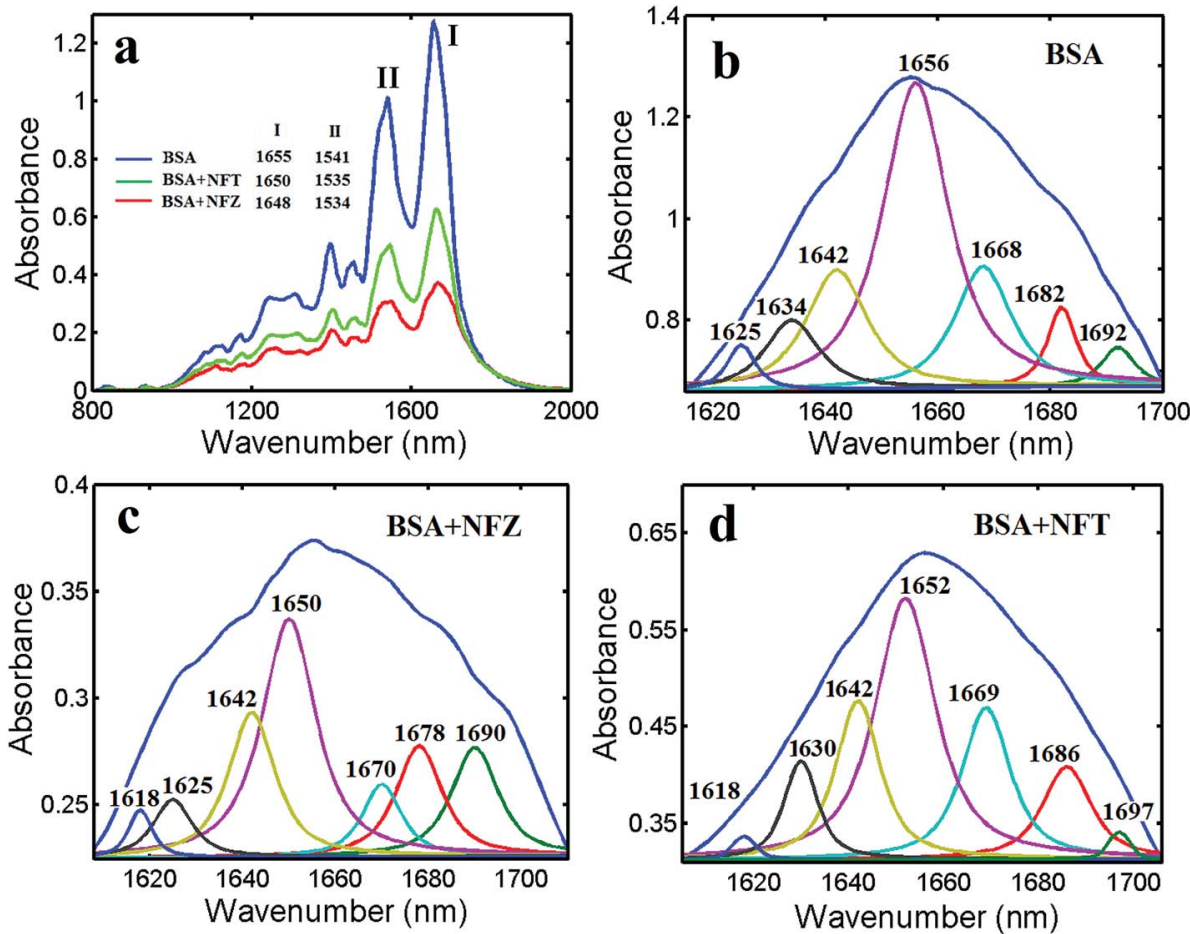

Fig. 3 (a) FT-IR spectra $\left(800-2000 \mathrm{~cm}^{-1}\right)$ of free BSA and NFZ/NFT-BSA complexes in Tris-Hcl buffer $\left(c_{\mathrm{BSA}}=2.5 \times 10^{-6} \mathrm{~mol} \mathrm{~L}{ }^{-1}\right.$, the molar ratio of NFZ/NFT to BSA is $4: 1)$; (b-d) curve-fitted amide I region $\left(1700-1600 \mathrm{~cm}^{-1}\right.$ ) of free BSA and its complexes with nitrofurans. 
and random coil structures suggest a partial protein unfolding at high nitrofurans concentrations.

\subsection{CD spectra analysis}

In the period of the interaction of nitrofurans with BSA, the changed in intramolecular or intermolecular forces, which remain the secondary or tertiary structures, might be affected, thus leading to further structure variations. ${ }^{33} \mathrm{CD}$ was conducted to predict the conformational changes in BSA induced through the binding of the nitrofurans.

Usually, in order to study the stereo structure of proteins, CD spectroscopy is prevalent in molecular biology study, particularly for secondary structure and variations of their conformation. CD spectroscopy supplies rapid evaluation of the conformational changes and measurements of protein secondary structure induced by ligand addition. ${ }^{34} \mathrm{~A}$ slight change in the near UV-CD spectra (250-320 nm) of BSA in the presence of NFZ/NFT was also observed (Fig. 2S, ESI $\dagger$ ). This suggested that NFZ/NFT induced the changes in the secondary and tertiary structure of the protein. In order to ascertain the secondary structural changes induced by NFZ/NFT, CD measurements in the far UV-CD region (200-250 nm) were carried out. Upon binding of ligand, intermolecular forces responsible for sustaining the secondary and tertiary structure may get rearranged, resulting in conformational alteration in the protein. Fig. 4 shows the CD spectra of free BSA and nitrofurans-BSA complex, and it demonstrated two negative bands at wavelengths of $208 \mathrm{~nm}$ and $222 \mathrm{~nm}$ in the UV region, which were both assigned to $\mathrm{n} \rightarrow \pi^{*}$ shifts of the peptide bonds in the $\alpha$-helix structure. The two wavelength points are the characteristic peaks of $\alpha$-helix structures and both of them can be used to calculate its content. Although the intensity of CD spectra was reduced with the addition of nitrofurans, the peaks did not shift obviously. If the $\mathrm{CD}$ result was manifested in line with MRE (mean residue ellipticity) in deg $\mathrm{cm}^{-2} \mathrm{dmol}^{-1}$, the following formula could be used:

$$
\operatorname{MRE}=\theta_{\text {obs }} /\left(10 n l c_{\mathrm{p}}\right)
$$

where $\theta_{\mathrm{obs}}$ is observed ellipticity, $c_{\mathrm{p}}$ is the molar concentration of the protein, $n$ is the amino acid residues number, $l$ is the path

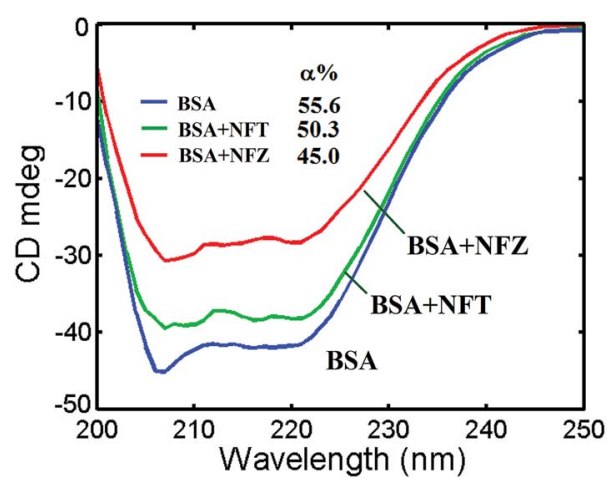

Fig. 4 Far UV-CD spectra of BSA alone and in the presence of NFZ and $\mathrm{NFT}\left(c_{\mathrm{BSA}}=2.5 \times 10^{-7} \mathrm{~mol} \mathrm{~L}^{-1}\right.$, the molar ratio of NFZ/NFT to BSA is $4: 1)$. length. The secondary structure percentage of $\alpha$-helix in BSA before and after the interaction with nitrofurans was determined by the following equations:

$$
\alpha-\text { Helix }=\left[\frac{[\theta]_{222 \mathrm{~nm}}+2340}{-30300}\right] \times 100 \%
$$

and $[\theta]_{222}$ is the MRE evaluated at wavelength of $222 \mathrm{~nm}, 2340$ value is the MRE of the random coil and $\beta$-form conformation cross at $222 \mathrm{~nm}$ and 30300 is the MRE of pure $\alpha$-helix at $222 \mathrm{~nm}$. Through the addition of nitrofurans, the intensities of the protein negative bands at 208 and $222 \mathrm{~nm}$ reduced gradually. The explanation may be that the stretched polypeptide structures of BSA caused by the combination of NFZ/NFT resulted in the exposure of the perturbation of the microstructures and hydrophobic cavities around the aromatic amino acid residues. Furthermore, the $\alpha$-helix content of BSA reduced with the additions of NFZ/NFT when the molar ratio of BSA/ nitrofuran was $1: 4$, which was in accordance with the results of the fluorescence and FT-IR spectra. From eqn (6) and (7), the quantitative analysis consequences of the $\alpha$-helix content were gained. A decrease of the $\alpha$-helix from $55.6 \%$ (free BSA) to $45.0 \%$ (NFZ-BSA $4: 1$ complex) and 50.3\% (NFT-BSA $4: 1$ complex) was obtained, indicating the reduction of $\alpha$-helix due to the interaction.

The results reveal that the impact of NFZ on the serum albumin conformational change is more remarkable than that of NFT, which prompts BSA unfold and adjust to a more incompact conformational status. The diverse effects of the two compound on the $\alpha$-helix structure of BSA could be associated with the configuration associative binding affinities. The NFZ/ NFT complexation could change the polypeptide structures of BSA that would then generate the beneficial spatial holes for the binding of BSA with NFZ/NFT. In addition, the CD spectra of BSA in the absence and presence of the nitrofurans were similar in shape, which indicating that the basic structure of BSA is conspicuously $\alpha$-helix even after bonding. ${ }^{35}$

\subsection{RLS and AFM analysis of the nitrofurans-BSA complex}

RLS is a sensitive method for investigation of aggregation of chromophores on biomacromolecules, and the RLS spectra of the nitrofurans-BSA system in Tris-HCl buffer ( $\mathrm{pH}$ 7.4) are shown in Fig. 3S. $\dagger$ It can be seen that both free BSA and free nitrofurans exhibits very vulnerable RLS signals, but when a settled concentration of NFZ and NFT was added with different amounts of BSA, the intensity of RLS were conspicuously increased. Basing on the theory of RLS, ${ }^{36}$ it can be deduced that RLS intensity is correlated to the dimension of the shaped particle and directly proportional to the square of molecular volume, and Chamani et al. ${ }^{37}$ have indicated that the larger particles could induce stronger light scattering signals. Therefore, the added NFZ and NFT possibly interacted with BSA in solution, and the formation of NFZ/NFT-BSA compound led to an increase of the RLS signal.

AFM was also executed in order to verdict the particle morphology and dimensions of the NFZ/NFT-BSA conjugates. BSA was observed to be adsorbed on the mica, the RMS (root 

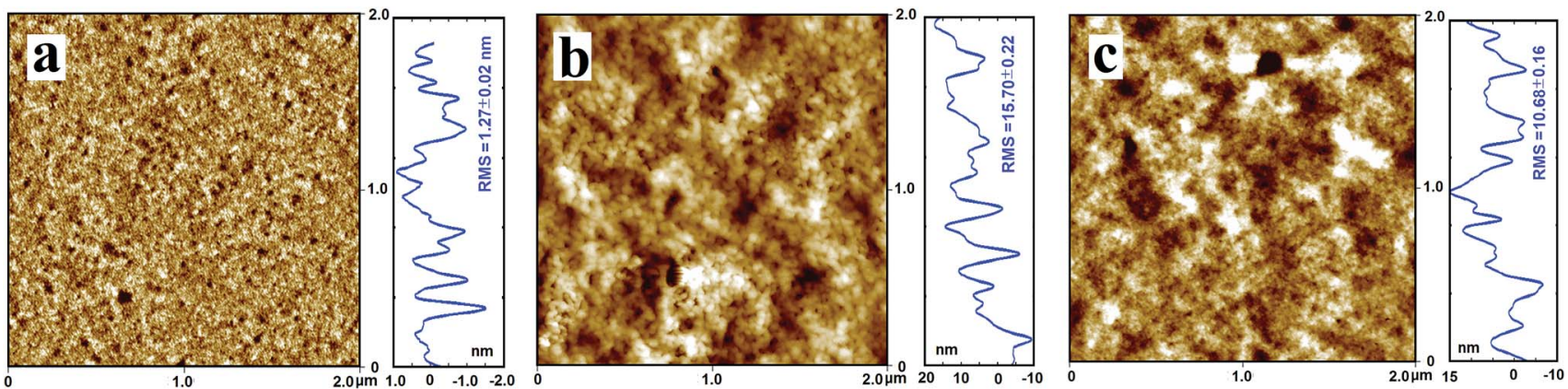

Fig. 5 AFM topography images of BSA and the NFZ/NFT-BSA complexes absorbed on mica with tapping mode in air (scan size of the image is $2 \mu \mathrm{m} \times 2 \mu \mathrm{m}, C_{\mathrm{BSA}}=6.67 \times 10^{-8} \mathrm{~mol} \mathrm{~L}^{-1}$, and the molar ratio of NFZ/NFT to BSA is $4: 1$ ).

mean square) roughness of the individual BSA molecule was found to be $1.27 \pm 0.02 \mathrm{~nm}$. The BSA molecule particle observed to be looser on the mica substrate upon interaction with NFZ/ NFT (Fig. 5). The RMS roughness of the molecules were changed to be $15.70 \pm 0.22 \mathrm{~nm}$ for the NFZ-BSA interaction and $10.68 \pm 0.16 \mathrm{~nm}$ for the NFT-BSA interaction, respectively. The change of the NFZ-BSA structure is greater than that of the NFT-BSA, which indicates that NFZ is more toxic than NFT.

The greater hydrodynamic radius of the conjugate particles indicates the formation of larger molecular weight polymers. In order to reduce the number of unpleasant factors to constitute a stable structure, the BSA molecular lessened the surface area in contact with water by molecular aggregation..$^{38}$ Hence, on the mica substrate we can see larger molecules, and this consequence also displayed that there were hydrophobic interactions between BSA and NFZ/NFT. ${ }^{39}$ The AFM result was accordance with the found of the FT-IR and CD and SFS spectra and confirmed the deduction from RLS experiment.

\subsection{Molecular docking studies}

BSA is a spherical protein constituting of a single peptide chain of 582 amino acids. It is composed of three analogical domains, each domain can be divided into two subdomains, A and B, which assembles to form heart shaped. The primary binding region on BSA is located in hydrophobic cavities in subdomains

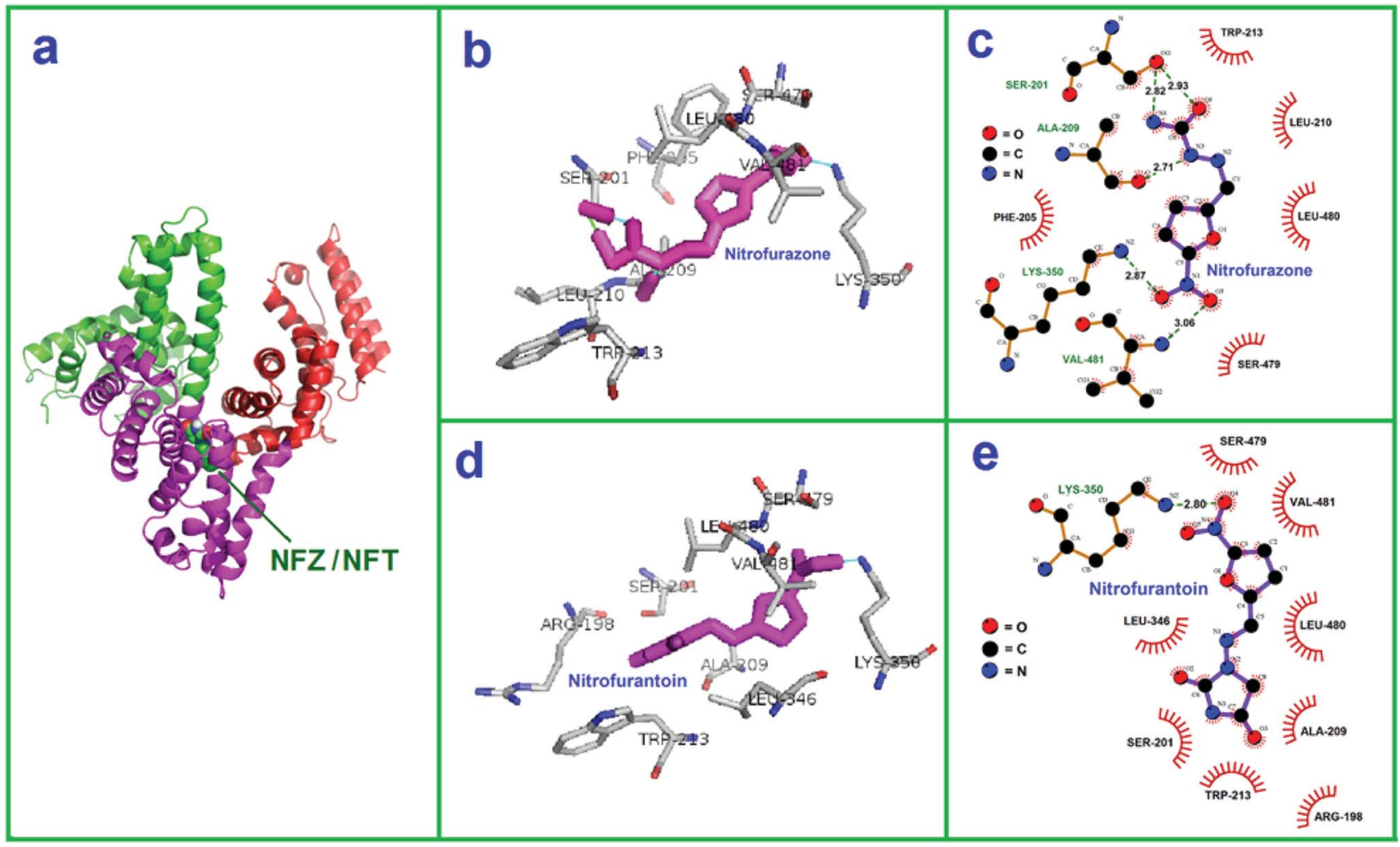

Fig. 6 Modeling of structure of BSA and nitrofurans. (a) Binding site of NFZ/NFT with BSA; (b) view of the docked NFZ binding in the hydrophobic pocket of subdomain IIA of BSA; (c) the hydrogen bond interaction (green dashed line) between NFZ and BSA. (d) View of the docked NFT binding in the hydrophobic pocket of subdomain IIA of BSA; (e) the hydrogen bond interaction (green dashed line) between NFT and BSA. 
IIA, which corresponds to the site $\mathrm{I}^{\mathbf{4 0}}$ According to Sudlow et al., the warfarin binds specifically to the site I whereas ibuprofen binds to site II of serum albumin. ${ }^{\mathbf{4 1}}$ Hence, in order to identify the binding site of NFZ/NFT on BSA, some extra experiments were done in this study. The concentration of BSA and warfarin/ ibuprofen were kept constant at $6.67 \times 10^{-8} \mathrm{~mol} \mathrm{~L}^{-1}$ and NFZ/NFT was added in the range of $0.00-2.13 \times 10^{-7} \mathrm{~mol} \mathrm{~L}^{-1}$, the binding constants (obtained from the double logarithmic equation) of NFZ-BSA/NFT-BSA were varied in presence of warfarin, which was less influenced by ibuprofen (Fig. 4S, Table 1S, in ESI $\dagger$ ). The binding constant of NFZ-BSA and NFT-BSA decreased from 4.27 $\times 10^{6} \mathrm{~L} \mathrm{~mol}^{-1}$ to $3.17 \times 10^{6} \mathrm{~L} \mathrm{~mol}^{-1}$ and $5.11 \times 10^{6} \mathrm{~L} \mathrm{~mol}^{-1}$ to $2.80 \times 10^{6} \mathrm{~L} \mathrm{~mol}^{-1}$ in the present of warfarin, respectively, which suggests the binding of NFZ/NFT to the site I.

The molecular modeling study was carried out to clearly describe the bioactivity mechanism and help us to better visual understanding the binding site on molecular level about the interaction between NFZ/NFT and BSA. See Fig. 6a, there is a large hydrophobic cavity in subdomain IIA to accommodate the NFZ/NFT, which indicates that the pattern of interaction is hydrophobic in essence because the binding site is located deeply inside the subject of protein instead of being on the surface of the BSA. ${ }^{42}$ The results revealed that there have hydrophobic amino acids Trp213, Leu210, Leu480, Phe205, Ala209, Val481 for NFZ and Leu480, Val481, Leu346, Ala209, Trp213 for NFT, indicating the existence of hydrophobic interaction between NFZ/NFT and BSA (Fig. 6b-e). There are hydrogen bonds interactions between the nitryl on the furan ring of NFZ/NFT and terminal amino group of the amino acids Lys-350. However, there are more hydrogen bonds interactions between NTZ and BSA, which further showed that NFZ is more vivacious than NFT when transported by BSA. What is more, there are also a number of weak electrostatic interactions between charged amino acids Ser479, Ser-201, Lys-350 with NFZ, Ser-479, Ser-201, Arg-198, Lys-350 with NFT, respectively. The results of molecular modeling manifested that the binding between NFZ/NFT and BSA was hydrogen binding and hydrophobic and electrostatic synergy, which was consistent with the above results.

It was found that the nitrofurans with a furan ring could interact with protein's hydrophobic residues. Generally, an electrostatic interaction could not be ruled out with respect to present findings, because BSA with an isoelectric point of 5.8, is negatively charged in Tris-HCl buffer of $\mathrm{pH}$ 7.4. For NFT, there is one $\mathrm{N}-\mathrm{H}$ and two carbonyl groups, and two five-membered heterocyclics, the greater steric hindrance from the structures, the less the possibility of hydrophobic interaction. Nitryl on the furan ring of NFZ/NFT was found to interact with the proteins hydrophobic residues to form hydrogen bonding. As a result, the apparent interaction between NFZ/NFT and BSA may be hydrogen binding.

\section{Conclusions}

The binding of two nitrofurans (NFZ and NFT) to BSA, has been systematically studied using fluorescence, FT-IR, CD spectroscopy and AFM. It was discovered that BSA recognized NFZ and NFT with different binding affinity, spectral response and structural perturbation. The fluorescence experimental results manifest that the quenching mechanism of BSA by NFZ/NFT is a static quenching procedure. The molecular simulation indicates that the hydrophobic forces and electrostatic interactions play dominant role during the ligand-protein binding process and effectively proves the binding of NFZ/NFT to BSA. The gained binding constants for NFZ/NFT with BSA are $10^{6} \mathrm{~L} \mathrm{~mol}^{-1}$, and the relatively high binding affinities manifest that the binding of NFZ/NFT to BSA might be specific. It has also found that the binding affinity of NFT to BSA was stronger than that of NFZ due to their diverse molecular structure. The FI-IR, CD and AFM showed that NFZ/NFT interact with both $\mathrm{C}=\mathrm{O}$ and $\mathrm{C}-\mathrm{N}$ groups of BSA, leading to the loss of $\alpha$-helix structure and the corresponding changes of some other secondary structures with different influence degrees. The impact on the conformational changes of BSA caused by NFZ is more conspicuous than that of NFT, which illustrates that NFZ is more toxic than NFT.

\section{Conflicts of interest}

There are no conflicts to declare.

\section{Acknowledgements}

The authors gratefully acknowledge the financial support of this study by the National Natural Science Foundation of China (NSFC-21665017), the State Key Laboratory of Food Science and Technology of Nanchang University (SKLF-KF-201606 and SKLFZZA-201612).

\section{References}

1 F. Kuralay and A. Erdem, Analyst, 2015, 140, 2876-2880.

2 M. M. Marshall, J. Ruzicka, O. K. Zahid, V. C. Henrich, E. W. Taylor and A. R. Hall, Langmuir, 2015, 31, 4582-4588.

3 A. Basu and G. S. Kumar, Food Chem., 2015, 175, 137-142.

4 A. Frostell-Karlsson, A. Remaeus, H. Roos, K. Andersson, P. Borg, M. Hamalainen and R. Karlsson, J. Med. Chem., 2000, 43, 1986-1992.

5 S. Bhuiya, A. B. Pradhan, L. Haque and S. Das, J. Phys. Chem. B, 2016, 120, 5-17.

6 S. Tunc, O. Duman, I. Soylu and B. K. Bozoglan, J. Hazard. Mater., 2014, 273, 36-43.

7 P. Thongsrisomboon, B. Liawruangrath, S. Liawruangrath and S. Satienperakul, Food Chem., 2010, 123, 834-839.

8 K. M. Cooper, J. V. Samsonova, L. Plumpton, C. T. Elliott and D. G. Kennedy, Anal. Chim. Acta, 2007, 592, 64-71.

9 N. Taokaenchan, T. Tangkuaram, P. Pookmanee, S. Phaisansuthichol, S. Kuimalee and S. Satienperakul, Biosens. Bioelectron., 2015, 66, 231-237.

10 S. E. Aiello, The Merck veterinary manual, Whitehouse Station, New Jersey, 2005.

11 H. Y. Zhai, L. C. Zhang, Y. F. Pan, K. S. Yuan, L. Huang and X. Yu, Chromatographia, 2015, 78, 551-556.

12 J. Points, D. T. Burris and M. J. Walker, Food Control, 2015, 50, 92-103. 
13 A. Kaufmann, P. Butcher, K. Maden, S. Walker and M. Widmer, Anal. Chim. Acta, 2015, 862, 41-52.

14 D. Kim, B. Kim, S. W. Hyung, C. H. Lee and J. Kim, J. Food Compos. Anal., 2015, 40, 24-31.

15 Y. B. Zhang, H. O. Qiao, C. Chen, Z. L. Wang and X. D. Xia, Food Chem., 2016, 192, 612-617.

16 T. Peter, All about albumin: Biochemistry Genetic and Medical Applications, Academic Press, San Diego, CA, 1996, pp. 9-75.

17 B. Tang, Y. M. Huang, X. L. Ma, X. X. Liao, Q. Wang, X. N. Xiong and H. Li, Food Chem., 2016, 212, 434-442.

18 Y. P. Wang, G. W. Zhang and L. H. Wang, J. Agric. Food Chem., 2015, 63, 75-84.

19 D. Wu, J. Yan, J. Wang, Q. Wang and H. Li, Food Chem., 2015, 170, 423-429.

20 X. Peng, G. W. Zhang, Y. J. Liao and D. M. Gong, Food Chem., 2016, 190, 207-215.

21 A. Sulkowska, J. Mol. Struct., 2002, 614, 227-232.

22 S. Huang, H. N. Qiu, S. Y. Lu, F. W. Zhu and Q. Xiao, J. Hazard. Mater., 2015, 285, 18-26.

23 T. T. Chen, X. T. Zhu, Q. Chen, M. Ge, X. P. Jia, X. Wang and C. W. Ge, Food Chem., 2015, 186, 292-297.

24 P. Mandal and T. Ganguly, J. Phys. Chem. B, 2009, 113, 14904-14913.

25 J. R. Lakowicz, Principles of Fluorescence Spectroscopy, Kluwer Academic/Plenum Publishers, New York, 2nd edn, 1999.

26 J. R. Lakowicz, Principles offluorescence spectroscopy, Plenum Press, New York, 3rd edn, 2006, p. 277.

27 T. P. Lakshmi, M. Mondal, K. Ramadas and S. Natarajian, Spectrochim. Acta, Part A, 2017, 183, 90-102.
28 P. D. Ross and S. Subramanian, Biochemistry, 1981, 20, 30963102.

29 D. M. Charbonneau and H. A. Tajmir-Riahi, J. Phys. Chem. B, 2010, 114, 1148-1155.

30 J. A. Pierce, R. S. Jackson, K. W. Vanevery, P. R. Griffiths and H. J. Gao, Anal. Chem., 1990, 62, 477-484.

31 S. N. Khan, B. Islam, R. Yennamalli, A. Sultan, N. Subbarao and A. U. Khan, Eur. J. Pharm. Sci., 2008, 35, 371-382.

32 P. Bourassa, C. D. Kanakis, P. Tarantilis, M. G. Pollissiou and H. A. Tajmir-Riahi, J. Phys. Chem. B, 2010, 114, 3348-3354.

33 A. S. Sharma and M. Ilanchelian, J. Phys. Chem. B, 2015, 119, 9461-9476.

34 Q. M. Sun, J. He, H. Q. Yang, S. S. Li, L. D. Zhao and H. Li, Food Chem., 2017, 233, 190-196.

35 Y. Liu, M. M. Chen, L. G. Jiang and L. Song, Food Chem., 2015, 181, 170-178.

36 J. Yang, X. D. Chen, R. W. Fu, W. A. Luo, Y. B. Li and M. Q. Zhang, Phys. Chem. Chem. Phys., 2010, 12, 2238-2245.

37 H. Iranfar, O. Rajabi, R. Salari and J. Chamani, J. Phys. Chem. $B, 2012$, 116, 1951-1964.

38 G. W. Zhang, L. Wang and J. H. Pan, J. Agric. Food Chem., 2012, 60, 2721-2729.

39 D. Y. Kim and W. S. Shin, Food Chem., 2015, 173, 1-6.

40 A. Bujacz, Acta Crystallogr., Sect. D: Biol. Crystallogr., 2012, 68, 1278-1289.

41 G. Sudlow, D. J. Birkett and D. N. Wade, Mol. Pharmacol., 1976, 12, 1052-1061.

42 M. Sarkar, S. S. Paul and K. K. Mukherjea, J. Lumin., 2013, 142, 220-230. 